\title{
Takotsubo Syndrome: A Literature Review
}

\author{
Sara Cristine Marques dos Santos ${ }^{1}$, Caio Teixeira Dos Santos ${ }^{1 *}$, Thais Lemos de Souza Macedo ${ }^{1}$, Raul \\ Ferreira de Souza Machado ${ }^{1}$, Maria Clara Carvalho da Costa $^{2}$ and Ivana Picone Borges de Aragão ${ }^{1}$
}

${ }^{1}$ Department of Medical Sciences, University of Vassouras, Brazil

${ }^{2}$ Department of Biomedicine Course, Veiga de Almeida University, Brazil

*Corresponding author: Caio Teixeira Dos Santos, Department of Medical Sciences, University of Vassouras, Brazil.

Received Date: September 03, 2019

Published Date: September 09, 2019

Resume

Takotsubo syndrome (TTS) or reversible left ventricular apical dysfunction [1] is characterized by symptoms like acute myocardial infarction (AMI), without evidence of coronary artery obstruction. There is evidence of systolic dysfunction of the left ventricle, with suggestive electrocardiographic alterations of AMI and elevation of spontaneously reversible cardiac enzymes. Occurs to exposure to physical or emotional stress and may lead to complications due to complete ignorance of its pathophysiology [2]. The aim of the present study is to seek a better understanding of this syndrome, based on previous research. A systematic literature review was conducted between 2005 and 2017 on Pubmed, Scielo and Medline search engines. The syndrome occurs predominantly in women, has a temporary character with good evolution and prognosis for the patient.

Keywords: Takotsubo Cardiomyopathy; Catecholamines; Ventricular Dysfunction; Cardiology

\section{Introduction}

Takotsubo syndrome (TTS) or reversible left ventricular apical dysfunction 1 is characterized by symptoms similar to acute myocardial infarction (AMI), without evidence of coronary artery obstruction. There is evidence of dysfunction itself Stolica the left ventricle, with ECG changes suggestive of AMI and elevated cardiac enzymes reversible spontaneously and may occur due to exposure to the physical or emotional stress, may present with complications due to total ignorance of its pathophysiology [2]. The aim of the present study is to seek a better understanding of this syndrome, based on previous research.

\section{Material and Methods}

A systematic review was conducted of the literature based on 15 articles between 2005 and 2017 in the languages: Portuguese and English, using the Pubmed database, Scielo, Medline, being used descriptors: Takotsubo cardiomyopathy, Ventricular Dysfunction and Electrocardiography.

\section{Literature Review}

Takotsubo cardiomyopathy or apical ballooning syndrome or left ventricular cardiomyopathy adre vigorous action is manifested in the form similar to AMI, the main differential diagnosis in the medical approach to emergency care service. Symptoms mimic AMI, however, with slight elevation markers of myocardial necrosis and absence of coronary artery obstruction [3]. This syndrome was initially identified and described in Japan and the name Takotsubo was given due to the similarity of its format with an artifact used to capture octopuses that the left ventricle presents during systole in the affected patient [4] Figure 1.

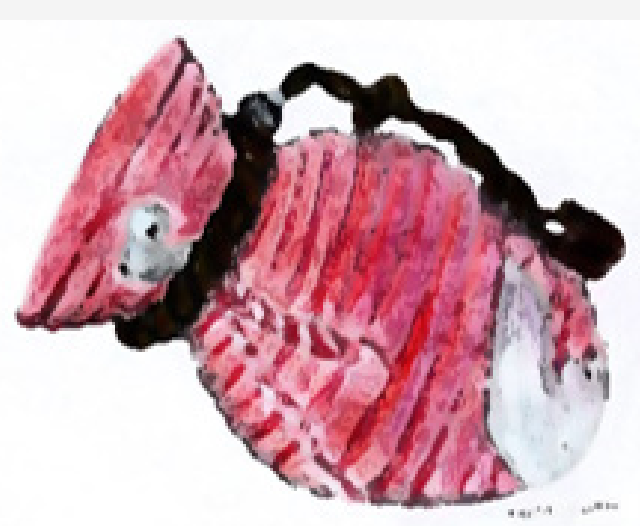

Figure 1: The left ventricle presents during systole in the affected patient. 
It is currently recognized by the American Heart Association and classified as a primary acquired cardiomyopathy, which means that it is only or mostly associated with the heart muscle [5]. There is a predominance in postmenopausal women (about $90 \%$ of cases). The occurrence is usually preceded by physical or emotional stress; however, the absence of a triggering event does not preclude the diagnosis of Takotsubo [4]. It can be considered a complication after cardiac surgery [3]. According to the Mayo Clinic, the criteria for the diagnosis of Takotsubo syndrome include the presence of segmental changes in the left ventricle with or without involvement of the apical region; absence of coronary obstruction or angiographic evidence of coronary plaque rupture; electrocardiographic abnormalities such as ST-segment elevation and / or T-wave inversion and subtle elevation of cardiac troponin are present; no diagnosis of pheochromocytoma or myocarditis [4]

In the blood analysis of the markers of myocardial necrosis in patients with TTS, they also demonstrated increased creatine kinase (CK-MB) in addition to troponin. Troponin T blood peak occurs at 24 hours, with gradual decrease, emphasizing that they are lower than those presented in patients with AMI [5-6]. Troponins I and $\mathrm{T}$ are biochemical biomarkers of myocardial necrosis [7]. Since they constitute an intracellular component, for release into the bloodstream, injury or death of myocardial cells is required.

B-type natriuretic peptide levels will be higher in patients with takotsubo than in those with myocardial infarction, and together with troponin $\mathrm{T}$, an important factor to help in the differential diagnosis between TTS and AMI [8]. In the electrocardiogram, the most common alteration is ST segment elevation 6 and also T wave inversion in precordial leads [9]. The magnitude of ST segment elevation and the number of leads with this pattern are generally lower in patients with TTS than in AMI [10]. It was observed that the values of interleukin - 6 (IL-6), an important factor in inflammation, and interleukin - 7 (IL-7), function in the homeostasis of cells lymphocytes, but can act in inflammatory processes, increases the STT during days hospitalization, while in patients with AMI, it decreases [11] . It was observed that the IL-6 is higher in patients with AMI at the time of hospitalization, tending throughout the hospitalization to be similar to that of patients with TTS. Left ventricular systolic dysfunction is temporary, with improvement of the clinical condition in a few days or weeks, generally with a good prognosis. Intrahospital mortality due to TTS is low, ranging from 1 to $2 \%$ 6. Research by the University Hospital of Zurich, with 25 cardiovascular centers based in nine different countries, enabled the international registration of creating Takotsubo (www.takotsuboregistry.com). We used data from patients diagnosed with TTS from 1998 to 2014, the diagnosis was made according to the Mayo Clinic criteria, previously mentioned in this article. Of the 1750 patients studied, $89.8 \%$ were women, $79.1 \%$ of them over 50 years old, being prevalent in postmenopausal women. Emotional factors such as triggers were more common in women and physical factors in men [12]. Physical factors include intracranial hemorrhage, sepsis, sexual intercourse, and pregnancy. Having as predisposing factors the postmenopausal period, comorbidity, mood changes, changes in the autonomic nervous system, among others [13]. The European Society of Cardiology has divided STT into two subtypes. Primary is described by patients who have acute episodes of cardiac symptoms, where they seek care in hospitals or with their doctor. There may or may not be associated stressors; You may have health conditions that may be considered risk factors but are not the primary cause of catecholamine increase. The secondary subgroup is where most cases occur in hospitalized patients where there is a sudden activation of the sympathetic nervous system or the increase of catecholamines, triggering the STT. Among the factors that may contribute to this event are respiratory, cardiological, neurological, psychiatric and post-surgery cases [14].

A recent study in a murine model demonstrated that infusion of high epinephrine concentrations may produce the characteristic reversible apical ballooning in the left ventricle associated with basal hypercontractility observed in TTS patients, reflecting the increased concentration of circulating catecholamines also increased at the myocardial level, as demonstrated in the acute phase [10]. Recurrence of TTS is rare, with an index of 0 to $10 \%$ of cases already registered, being more common in younger women, and more careful follow-up is advised in this group of patients. It was not possible to identify a factor responsible for this recurrence as well as a therapeutic plan for prevention has not been determined [15].

\section{Final Considerations}

The STT by present clinically similar to AMI, need to be investigated as a differential diagnosis by the team responsible for the service. It occurs predominantly in women and is considered temporary and with good patient outcome. To help diagnose the measurement of interleukins and cardiac enzymes are a great ally. It has a rare recurrence in patients with a better prognosis than in AMI, in which coronary artery blockade is involved as a triggering factor for its occurrence.

\section{Acknowledgement}

None.

\section{Conflict of Interest}

No conflict of interest.

\section{References}

1. Sousa José Marconi Almeida De, Knobel M, Buchelle G, Sousa José Augusto M De, Fisher Claudio H, et al. (2005) Reversible apical dysfunction syndrome (Takotsubo). Arq Bras Cadriel [Internet] 84(4): 340-342.

2. Nobrega S, Brito D (2012) Takotsubo cardiomyopathy: state of the art. Rev Port Cardiol [internet] 31(9): 589-596.

3. Kogan A, Ghosh P, Schwammenthal, Raanani E (2018) Takotsubo Syndrome After Cardiac Surgery. Ann Thorac Surg [internet] 85: 14411443.

4. Scantlebury DC, Prasad A (2014) Diagnosis of takotsubo cardiomyopathy Mayo Clinical criteria Circulation Journal 78 (9): 2129-2139.

5. BJ Maron, JA Towbin, Thiene G, Antzelevitch C, Corrado D, et al. (2006) Contemporary Definitions and Classification of the Cardiomyopathies. Circulation, [online] 14: 1807-1816. 
6. Prasad A, Lerman A, Rihal CS (2008) Apical ballooning syndrome (Tako-Tsubo or stress cardiomyopathy): A mimic of acute myocardial infarction. American Heart Journal 155 (3): 408-417.

7. Martins Carla Sofia (2009) Troponin: Structure, Pathophysiology and Clinical Importance Beyond Myocardial Ischemia. File Med [Internet] 23 (6): 221-240

8. Madhavan M, Borlaug BA, Lerman A, Rihal CS, Prasad A, et al. Stress hormone and circulating biomarker profile of apical ballooning syndrome (Takotsubo cardiomyopathy): insights into the clinical significance of B-type natriuretic peptide and troponin levels. Heart 95: 1436-1441.

9. Crespo R, Paulino R, Bernardo R, Raposo N (2012) The role of electrocardiogram in the diagnosis of Takotsubo cardiomyopathy. ESSCVP Health Sciences Rev [internet] 4: 6-13.

10. Pelliccia F, Kaski JC, Crea F, Camici PG (2017) Pathophysiology of Takotsubo Syndrome. Circulation V. 135(24).
11. Pirzer R, Elmas E, Haghi D, Lippert C, Kralev S, et al. (2012) Platelet and monocyte activity markers and mediators of inflammation in Takotsubo cardiomyopathy. Heart Vessels 27: 186-192.

12. Templin C, Ghadri JR, Diekmann J, Napp LC, Bataiosu DR, et al. (2015) Clinical features and outcomes of takotsubo (stress) cardiomyopathy. New England Journal of Medicine 373(10): 929-938.

13. Y-Hassan S, Tornvall P (2017) Epidemiology, pathogenesis, and management of takotsubo syndrome. Clinical Autonomic Research: Official Journal of the Clinical Autonomic Research Society 28 (1): 53-65.

14. Lyon AR, Bossone E, Schneider B, Sechtem U, Citro R, et al. (2016) Current state of knowledge on takotsubo syndrome: a Position Statement from the Taskforce on takotsubo Syndrome of the Heart Failure Association of the European Society of Cardiology. Eur J Heart Fail 18: 8-27.

15. Kato K, Kitahara H, Kobayashi Y (2016) Recurrence of Takotsubo Cardiomyopathy. International Cardiovascular Forum Journal v. 5. 